\title{
Oral rehabilitation of a patient with li-fraumeni syndrome: a clinical case report
}

\begin{abstract}
Li-Fraumeni syndrome (LFS) is a rare condition that predisposes patients and their families to develop various cancers, such as sarcoma, breast cancer, brain tumors, leukemia, and adrenocortical carcinoma, typically at early ages. The syndrome is associated with a germline mutation of the tumor suppressor gene TP53. Early detection of LFS is key to successful treatment and survival for these patients. This case report describes the diagnosis and oral rehabilitation of a patient with LFS who developed osteosarcoma in the left anterior maxilla that descended into the anterior maxillary sinus wall and into the nose.
\end{abstract}

Volume 10 Issue 2 - 2019

\author{
Ahmad Qazali,' Hazem Alharbi,' Theresa \\ Hofstede ${ }^{2}$ \\ 'Department of Prosthodontics, Tiabah University, Saudi Arabia \\ ${ }^{2}$ Associate Professor, Department of Head and Neck Surgery, \\ Division of Surgery, The University of Texas MD Anderson \\ Cancer Center, Houston Texas, USA
}

\author{
Correspondence: Theresa Hofstede, The University of Texas \\ MD Anderson Cancer Center, 1515 Holcombe Blvd, Unit \\ Number: 1445, Houston, TX 77030, USA, \\ Email ahmado_25@hotmail.com
}

Received: February 28, 2019 | Published: March 06, 2019

\section{Introduction}

Li-Fraumeni syndrome (LFS) is a rare condition that predisposes patients and their families to develop various neoplasms, typically at early ages. The syndrome has an autosomal dominant pattern of inheritance and is associated with a germline mutation of the tumor suppressor gene TP53., ${ }^{1,2}$ Common among these neoplasms are early-onset sarcomas of bone and soft tissues, carcinomas of the breast and adrenal cortex, brain tumors, and acute leukemias. ${ }^{1}$ In addition to these, a large range of other malignant neoplasms have been reported in TP53 mutation carriers, including bronchoalveolar, pancreatic, gastric, ovarian, and colorectal cancers. ${ }^{3-5}$ The classic clinical representation of LFS is an individual who was diagnosed as having a sarcoma before the age of 45 years, with a first-degree relative with any cancer diagnosed before he or she was age 45 , and another first-degree or second-degree relative with either a sarcoma diagnosed when the patient was at any age or any other type of cancer diagnosed before the patient reached age 45..$^{2,4}$ The phenotypes of families with TP53 mutations can vary, due to lifestyle or due to the genetic background of the genes involved in the $\mathrm{p} 53$ pathway. ${ }^{6,7}$ About $70 \%$ of families meet the classic characteristics of LFS, ${ }^{8,9}$ including about $40 \%$ who meet the diagnostic criteria for Li-Fraumeni-like syndrome $(\mathrm{LFL})^{10}$ and between $7 \%$ and $20 \%$ who are considered to have de novo mutations. ${ }^{11}$ The cumulative cancer risk for patients with LFS is approximately $50 \%$ by age 40 years and up to $90 \%$ by the age of $60 .{ }^{12}$ Women are at higher risk than men. ${ }^{13,14}$ Apart from the likelihood of being affected by a number of neoplasms, individuals with LFS also have a high risk of multiple primary cancers. ${ }^{5,15-17}$ This case report describes the dental treatment of a patient with LFS and may provide guidance for the oral treatment of other patients with cancer predisposition syndromes.

\section{Clinical report}

\section{Clinical history}

A 23-year-old man presented to the Department of Head and Neck Surgery at The University of Texas MD Anderson Cancer Center with slowly growing facial swelling. The patient had first noticed this swelling in May 2015. An oral and maxillofacial surgeon (private practitioner) confirmed osteosarcoma after a biopsy. The patient had been treated by the surgeon a few times previously. He also had a history of unclassified high-grade spindle cell sarcoma of the right shoulder, which was excised several years previously, followed by excision of an angiolipoma, also in his right shoulder, a few years later. Computed tomographic (CT) imaging revealed a predominantly exophytic $33 \times 17 \mathrm{~mm}$ mass, stemming from the left anterior maxilla with aggressive periosteal reaction and an associated soft tissue component. This mass was significantly larger than its previously measured $27 \mathrm{~mm}$ transverse diameter, measured 3 months previously. Sclerotic changes were also observed within the left anterior maxillary alveolar ridge. The absence of metastatic features or focal parotid or facial lymphadenopathy, despite bilaterally prominent lymph nodes in the upper neck, was noticed at the time of the scan (Figure 1).

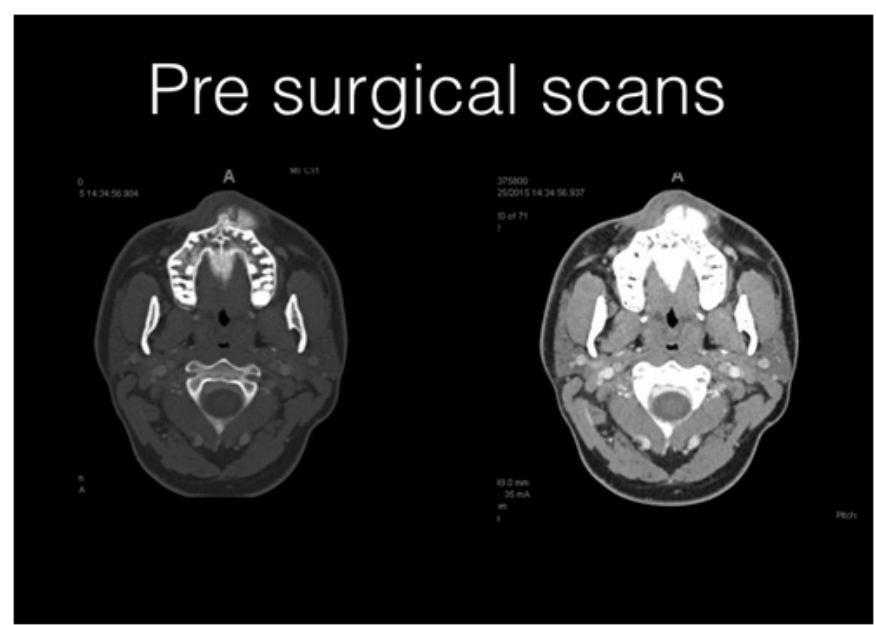

Figure I Presurgical computed tomographic (CT) scans, axial view.

\section{Family history}

The patient reported that none of his living family members had a history of cancer. These included, on his father's side, his 46-yearold father, 39-year-old paternal uncle, and paternal grandparents in their 70s; included on his mother's side were his 45-year-old mother, maternal uncles in their $40 \mathrm{~s}$, two maternal half aunts in their $50 \mathrm{~s}$, and maternal grandparents in their 70s. Furthermore, his 17- and 10-yearold younger sisters had no history of cancer. 


\section{Genetic testing}

Genetic testing was performed at MD Anderson, and the results indicated that the patient had a gene TP53 mutation. The mutation was believed to possibly be de novo, since none of the patient's living relatives tested at MD Anderson showed positive results. These included both parents, who tested negative for gene mutations. The patient's sisters had had no genetic testing done at the time this report was written. The patient was informed about the possible future occurrence of various core cancers, leukemia, and other childhood cancers in individuals with LFS. The patient was advised to undergo regular annual cancer surveillance, such as magnetic resonance imaging (MRI) scans, throughout his lifetime and to avoid exposure to radiation, including radiation therapy, to reduce the risk of radiationrelated malignancy.

\section{Oral and dental management}

Clinical and radiographic findings revealed that the patient had a tumor resting on the left anterior maxilla descending into the anterior maxillary sinus wall and into the nose. It did not involve the nasal mucosa, and very little hard palate was involved. The patient underwent left infrastructure maxillectomy with resection of the nasal floor and inferior maxillary sinus (Figures $2 \& 3$ ). Immediately after the surgery, the patient was rehabilitated with a maxillary acrylic resin obturator to restore function, esthetics, speech, and lip support. One week after surgery, the prosthesis was changed to an interim acrylic resin obturator. The patient was followed up regularly until healing, and the obturator was modified as necessary. As the healing progressed, the edges of the wound, including the upper lip, showed

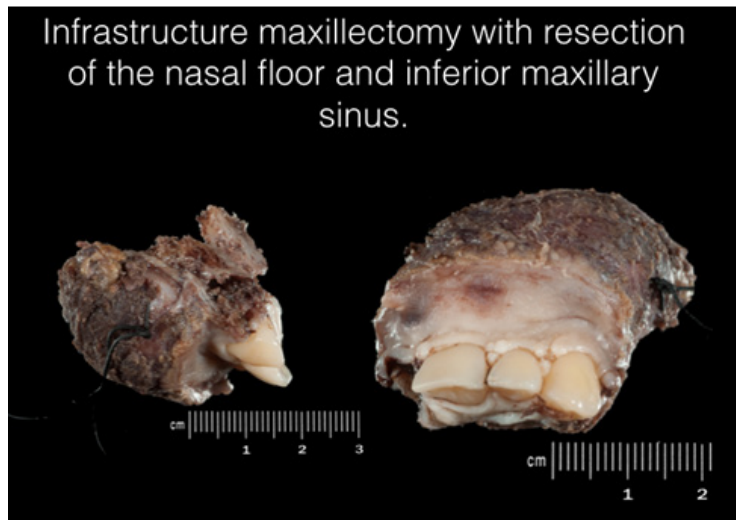

Figure 2 Resected tumors involving the nasal and maxillary sinus floor.

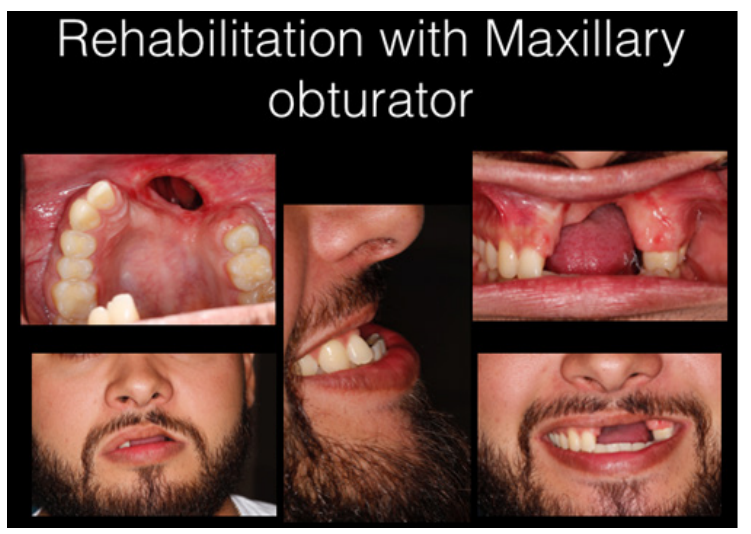

Figure 4 Postsurgical clinical photos of the maxillary defect. progressive retraction. This resulted in the prosthesis becoming slightly loose and the lip support becoming inadequate. The bulb of the obturator prosthesis was relined with a soft liner (Trusoft, Bosworth, USA), accordingly, to provide adequate support and retention. Once the wound was completely healed and no further retraction observed (4 months after surgery), the process for fabrication of the definitive obturator was started (Figure 4). An irreversible hydrocolloid (Supergel Alginate, Gibbstown, NJ, USA) primary impression was made with use of a stock metal tray. The cast was poured using type IV gypsum (Resin Rock, Patterson Dental, Bothell, Wshington, USA). The undesirable undercuts were blocked out with wax in the primary cast, and an acrylic resin custom tray (Triad TrueTray, Dentsply, York, Pennsylvania, USA) was fabricated. A final impression was made with modelling plastic (Impressio compound, Kerr, Orange, California, USA) border molding and poly-vinyl siloxane (PVS) wash impression (Aquasil Ultra, Dentsply). A cast metal removable partial denture prosthesis framework was fabricated and tried in the patient's mouth, and vertical and centric relations were established in a customary manner with the wax rim record base. Once the teeth were tried in and approved, hollow definitive obturator prosthesis was fabricated by using heat-processed acrylic resin. On delivery, the resin extensions into the undercuts were relieved. Pressure-indicating paste and disclosing wax were used to identify the excessive tissue displacement and were adequately relieved (Figure 5). The superior surface of the obturator was rounded and lightly polished with fine pumice. The prosthesis had good stability and retention, and the patient maintained the same lifestyle as before the procedure (Figure $6)$.

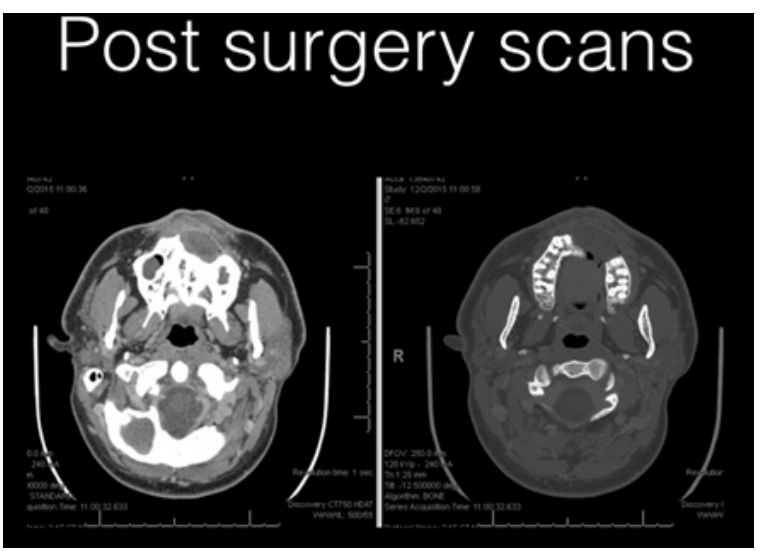

Figure 3 Postsurgical computed tomographic (CT) scans, axial view.

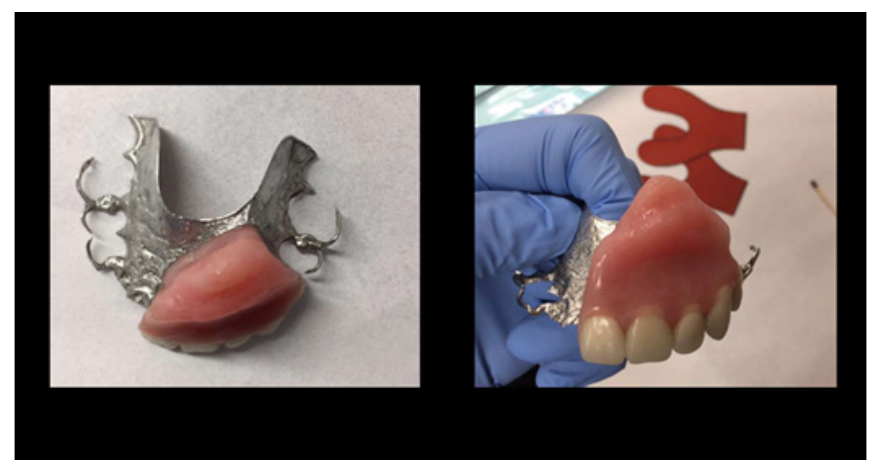

Figure 5 Extraoral view of definitive obturator. 


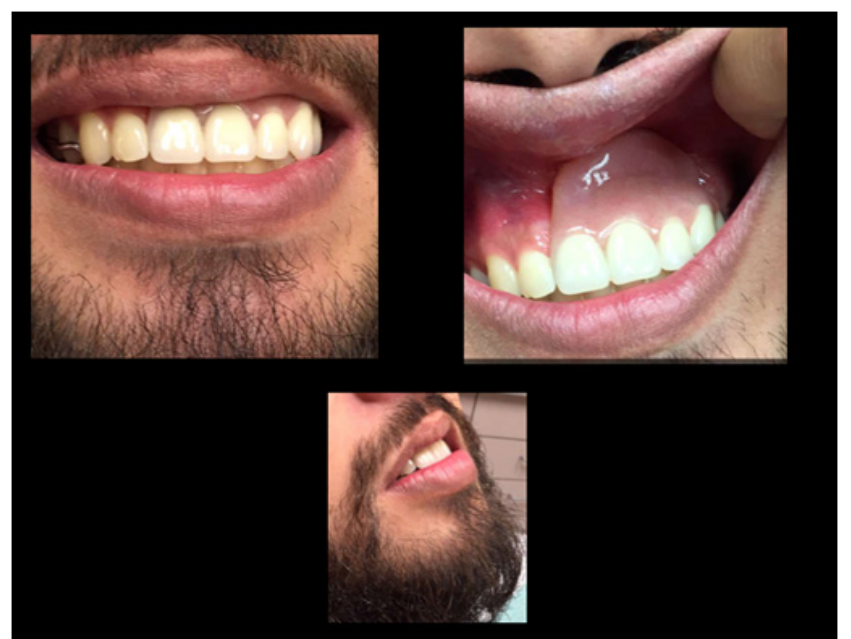

Figure 6 Intraoral view of definitive obturator.

\section{Discussion}

The above case report describes a 23 -year-old male patient who was diagnosed as having Li-Fraumeni syndrome. The patient's genetic testing indicated a TP53 gene mutation, and he presented with a history of high-grade spindle cell sarcoma of the right shoulder, which conformed to the classic clinical representation of a sarcoma developing before the age of 45 years. ${ }^{2,3}$ However, none of the other classic clinical representation characteristics were seen, especially in the familial phenotypical and genetic background. ${ }^{2,3,6}$ Therefore, the patient was considered to have a de novo gene mutation, which is reported in the literature in only $7 \%-20 \%$ of cases. ${ }^{7,8}$ It has been variously reported in the literature that by the third to fourth decade of life, approximately $50 \%$ of the TP53 mutation carriers develop cancer. ${ }^{9,10,15-17}$ Females are at a higher lifetime risk at close to $100 \%$, compared with males who have a $70 \%$ risk. ${ }^{10,11,15}$ In general, the high predisposition of patients with LFS to cancer as well as multi-organ tumorigenesis is associated with substantial morbidity and high mortality. ${ }^{3,6,15-17}$ This presents a particular challenge for programs involved with cancer risk management and treatment. In addition, an urgent need exists for enhanced clinical recognition of these syndromes so that LFS can be diagnosed and so patients at risk of this disease can be referred to suitable multidisciplinary teams for ongoing systemic and oral management, including genetic testing and counselling, psychological and familial counselling, tumour surveillance, cancer risk management, and appropriate cancer care. ${ }^{11-17}$

\section{Summary}

This case report describes the diagnosis and oral rehabilitation of a patient with LFS who developed osteosarcoma in the left anterior maxilla that descended into the anterior maxillary sinus wall and into the nose. A cast metal removable partial denture prosthesis framework was fabricated to restore the patient's function and appearance and maintaining his life style.

\section{Acknowledgments}

None.

\section{Conflicts of interest}

The author declares that there is no conflict of interest.

\section{References}

1. Nichols KE, Malkin D, Garber JE, et al. Germ-line p53 mutations predispose to a wide spectrum of early-onset cancers. Cancer Epidemiol Biomarkers Prev. 2001;10(2):83-87.

2. Li FP, Fraumeni JF, Mulvihill JJ, et al. A cancer family syndrome in twenty-four kindreds. Cancer Res. 1988;48(18):5358-5362.

3. Mathew B Yugelun, Serena Masciari, Victoria A Joshi. Germline TP53 Mutations in Patients With Early-Onset Colorectal Cancer in the Colon Cancer Family Registry. JAMA Oncol. 2015;1(2):214-221.

4. Malkin D. Li-fraumeni syndrome. Genes Cancer. 2011;2(4):475-484.

5. Gonzalez KD, Noltner KA, Buzin CH, et al. Beyond Li Fraumeni Syndrome: clinical characteristics of families with p53 germline mutations. J Clin Oncol. 2009;27(8):1250-1256.

6. Merino D, Malkin D. p53 and hereditary cancer. Subcell Biochem. 2014;85:1-16.

7. McBride KA, Ballinger ML, Killick E, et al. Li-Fraumeni syndrome: cancer risk assessment and clinical management. Nat Rev Clin Oncol. 2014;11(5):260-271.

8. Varley JM. Germline TP53 mutations and Li-Fraumeni syndrome. Hum Mutat. 2003;21(3):313-320.

9. Bouaoun L, Sonkin D, Ardin M, et al. TP53 Variations in Human Cancers: New Lessons from the IARC TP53 Database and Genomics Data. Hum Mutat. 2016;37(9):865-876.

10. Birch JM, Hartley AL, Tricker KJ, et al. Prevalence and diversity of constitutional mutations in the p53 gene among $21 \mathrm{Li}$-Fraumeni families. Cancer Res. 1994;54(5):1298-1304.

11. Gonzalez $\mathrm{KD}$, Buzin $\mathrm{CH}$, Noltner $\mathrm{KA}$, et al. High frequency of de novo mutations in Li-Fraumeni syndrome. J Med Genet. 2009;46(10):689-693.

12. Lustbader ED, Williams WR, Bondy ML, et al. Segregation analysis of cancer in families of childhood soft-tissue-sarcoma patients. Am J Hum Genet. 1992;51(2):344-356.

13. Wu CC, Krahe R, Lozano G, et al. Joint effects of germ-line TP53 mutation, MDM2 SNP309, and gender on cancer risk in family studies of Li-Fraumeni syndrome. Hum Genet. 2011;129:663-673.

14. Fang S, Krahe R, Bachinski LL, et al. Sex-specific effect of the TP53 PIN3 polymorphism on cancer risk in a cohort study of TP53 germline mutation carriers. Hum Genet. 2011;130(6):789-794.

15. Mai PL, Best AF, Peters JA, et al. Risks of first and subsequent cancers among TP53 mutation carriers in the National Cancer Institute LiFraumeni syndrome cohort. Cancer. 2016;122(23):3673-3681.

16. Chompret, A. et al. p53 germline mutations in childhood cancers and cancer risk for carrier individuals. Br J Cancer. 2000;82(12):19321937.

17. McBride KA, Ballinger ML, Killick E, et al. Li-Fraumeni syndrome: cancer risk assessment and clinical management. Nat Rev Clin Oncol. 2014;11(5):260-271. 